\title{
Basic Study on Expressway Congestion Mitigation using Cellular Automata
}

\author{
Kodai Miyamoto, Taketo Kamasaka, Makoto Sakamoto, Tsunehiro Yoshinaga
}

\begin{abstract}
Traffic congestion has become a serious social problem in Japan. In particular, traffic congestion causes enormous time and economic losses on expressways, which are intended to facilitate smooth traffic flow. It also causes environmental problems and a decrease in logistics efficiency, so efforts to eliminate or reduce traffic congestion are essential. The elimination and mitigation of traffic congestion on highways is a factor in reducing traffic accidents and fatalities. In recent years, with the improvement of computing capabilities, research on traffic congestion reduction and mitigation has been conducted using traffic simulation. Traffic simulation allows us to reproduce and evaluate traffic phenomena that occur on roads on a computer. The Kakuto Tunnel is located on the border between Ebino City in Miyazaki Prefecture and Hitoyoshi City in Kumamoto Prefecture. The Kakuto Tunnel has insufficient traffic capacity and is frequently congested on holidays. In this study, we created a traffic simulator assuming the Kakuto Tunnel. In the simulation, we examined the effectiveness of increasing the number of lanes as a method of reducing traffic congestion.
\end{abstract}

Keywords: cellular automaton, comlex system, expressway, Kakuto Tunnel, traffic congestion.

\section{INTRODUCTION}

Currently, traffic congestion is a social problem in Japan. The annual time lost in traffic congestion in Japan is about 3.8 billion hours, and the economic loss is about 12 trillion yen [1]. Traffic congestion causes enormous time and economic losses, especially on expressways, which are designed for smooth traffic. In addition, it causes environmental problems due to automobile exhaust emissions and a decrease in the efficiency of logistics, so efforts to eliminate and alleviate traffic congestion are essential. On expressways, there are places where traffic congestion tends to occur. It has been shown that speed reduction tends to occur on uphill slopes and in tunnels, and that traffic jams tend to occur there [2]. In order to eliminate or alleviate traffic congestion, it is necessary to improve

Manuscript received on September 14, 2021.

Revised Manuscript received on September 18, 2021.

Manuscript published on September 30, 2021.

* Correspondence Author

Kodai Miyamoto*, Department of Computer Science, Engineering, Miyazaki University, Miyazaki, Japan. Email: hm16043@student.miyazaki-u.ac.jp

Taketo Kamasaka, Department of Computer Science, Engineering, Miyazaki University, Miyazaki, Japan. Email: hm16011@student.miyazaki-u.ac.jp

Makoto Sakamoto, Department of Computer Science, Engineering, Miyazaki University, Miyazaki, Japan. Email: fruits2000jp@yahoo.co.jp

Tsunehiro Yoshinaga, Department of Computer Science and Electronics Engineering, Tokuyama College of Technology, Yamaguchi, Japan. Email: fruits2000jp@yahoo.co.jp

(C) The Authors. Published by Blue Eyes Intelligence Engineering and Sciences Publication (BEIESP). This is an open access article under the CC BY-NC-ND license (http://creativecommons.org/licenses/by-nc-nd/4.0/) roads and create a stable flow of vehicles. However, improving an expressway requires a great deal of time and labor, as it requires analysis of traffic conditions through field surveys and restrictions through construction.

Recently, traffic simulators have been used for traffic analysis. A traffic simulator is a tool that can reproduce traffic phenomena on a road on a computer and evaluate them. Traffic simulators can save a lot of time and effort because they do not require a trip to the site. Traffic simulations in land transportation are mainly used for traffic jam countermeasures, traffic flow analysis, and road maintenance planning. There is a long tunnel called Kakuto Tunnel between Ebino City in Miyazaki Prefecture and Hitoyoshi City in Kumamoto Prefecture. In this study, traffic simulation is conducted assuming the Kakuto Tunnel. A simple simulator will be created using a simple and computationally inexpensive cellular automaton model [3]. In the simulation, we will examine whether increasing the number of lanes is an effective way to reduce traffic congestion.

\section{CURRENT STATUS OF TRAFFIC CONGESTION ON HIGHWAYS}

This chapter explains the definition of traffic congestion on expressways, and describes the current status of traffic congestion throughout Japan and in Miyazaki Prefecture.

\section{A. Defining Congestion on Highways}

A traffic jam on an expressway is defined as a situation in which vehicles are traveling at a low speed of $40 \mathrm{~km} / \mathrm{h}$ or less, or repeatedly stopping and starting, for a distance of $1 \mathrm{~km}$ or more and for a duration of 15 minutes or more [2].

\section{B. Current Traffic Congestion in Japan}

Currently, traffic congestion on ordinary roads is caused by traffic concentration during morning and evening commuting hours, accidents, and construction work. Traffic congestion can also be caused by where people park on the road. The three main causes of traffic congestion on Japanese highways are "traffic concentration," "construction congestion," and "accident congestion" [2]. Traffic concentration congestion is caused by the concentration of traffic beyond the traffic capacity set when the road was designed. Construction congestion is caused by construction regulations, and accident congestion is caused by traffic accidents.

- Traffic congestion

Traffic congestion accounts for about $64 \%$ of all congestion in Japan [2]. On expressways, there are certain areas where traffic congestion is likely to occur due to traffic concentration.

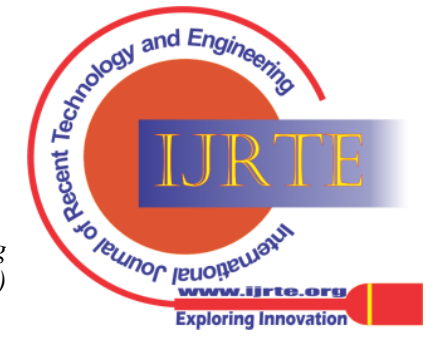




\section{Basic Study on Expressway Congestion Mitigation using Cellular Automata}

It has been shown that congestion is more likely to occur on uphills, sags, and tunnels [2]-[4]. A place where a slope changes from downhill to uphill is called a sag area. On an uphill slope or sag section, if some vehicles do not notice the decrease in speed, the distance between them and the following vehicles shrinks, causing the following vehicles to brake one after another, resulting in a traffic jam. As a countermeasure to traffic congestion on uphill slopes, LED indicator boards are installed to make drivers aware of the need to increase speed [4]. In tunnels, the driver's speed decreases due to darkened visibility and a sense of pressure, and as in uphill traffic, congestion is caused by braking by following vehicles. As a countermeasure against traffic congestion in tunnels, speed reduction is controlled by improving the visibility environment [4]. In bad weather, the power may be cut off and the ventilation system may stop. If traffic congestion occurs in that environment, drivers may develop carbon monoxide poisoning due to vehicle exhaust and lack of oxygen content. In addition, if the driver spends a economy class syndrome, which is dangerous.

\section{Current Status of Transportation in Miyazaki Prefecture}

In Miyazaki Prefecture, the number of cars owned is about 900 per 1,000 people "Fig. 1" [5]. This is a high figure compared to the rest of Japan.

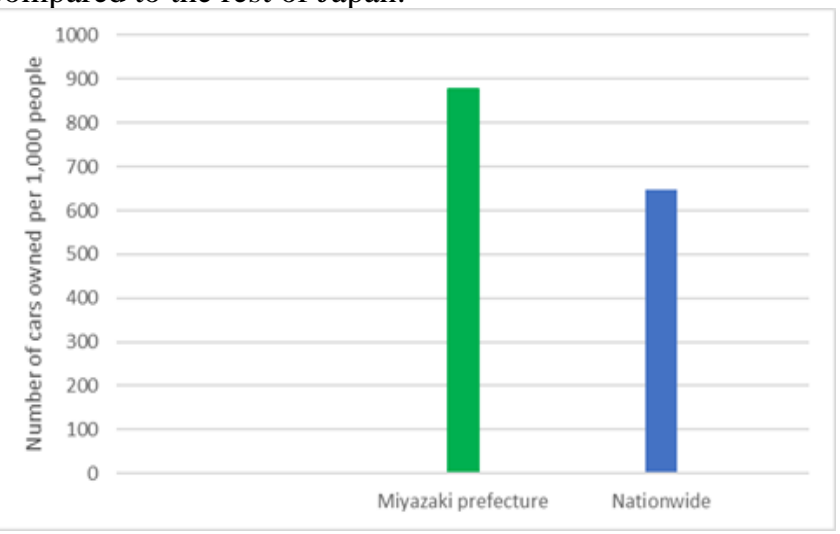

Fig. 1. Number of cars owned in Miyazaki Prefecture compared to the rest of Japan.

With a driver's license ownership rate of approximately $70 \%$, automobiles are an essential part of daily life for the people of Miyazaki Prefecture [8].

In rural areas, road maintenance is lagging behind in both general roads and highways. Road maintenance has the benefit of improving traffic flow and safety for pedestrians and cyclists. Especially for expressways, the traffic capacity at the time of design is often applied to roads that have not been maintained for many years.

Currently, many roads in Miyazaki Prefecture have inadequate traffic capacity.

\section{TRAFFIC SIMULATION METHODS}

The models used to represent traffic flow can be roughly divided into the following two types

- Macroscopic models [3]-[6].

The flow of vehicles is viewed as a fluid, not as individual vehicles. It is a model that focuses on changes in the density of vehicles on a road. It can be used to simulate a large area such as a large-scale road network. It is mainly applied when long time in the car in a traffic jam, he or she may develop

you want to understand the traffic flow roughly. The macromodel is sometimes referred to as a fluid model.

- Microscopic model [3]-[6].

It simulates the movement of individual cars. It can capture the deceleration of a car due to the influence of the car in front. It is often used to simulate a relatively small area such as a small road network. It can be used to simulate changes in traffic flow due to the addition of a lane at an intersection. The micro model is sometimes called a follow-up model. The cellular automaton model is included in the micro model.

In this study, the micro model is applied to perform a dynamic simulation limited to a long tunnel in Miyazaki Prefecture.

\section{A. Development environment}

Cellular automata were proposed by John von Neuman and Stanisław Marcin Ulam in the early 1950s [3]. A lattice of cells and simple rules can be used to represent complex phenomena.

Stephen Wolfram studied the way one-dimensional cellular automata evolve in time, and classified the patterns themselves into four features [3].

- uniform patterns

Quick, stable, and homogeneous in all initial states.

- periodic pattern

Quickly stable or periodically oscillating state in all initial states.

- chaotic pattern

A state in which the entire cell keeps changing randomly in all initial states.

- complex pattern

Regular and random patterns coexist to form a complex pattern.

One of the micro-models, the cellular automaton model, can be applied to traffic simulation to simplify the traffic flow.

\section{B. Rule 184}

In the cellular automata model, we introduce a basic model called Rule 184. Rule 184 is one of the rules of one-dimensional cellular automata [3]. In traffic simulations, it can be used as a simple model of traffic flow on a one-lane highway. The state of a cell in rule 184 has only 0 and 1 . In addition to its position itself, the next state is determined by the cells on the left and right. The motion of the car in this study is represented by vertical or horizontal movement only. It does not move diagonally or by skipping multiple cells.

Rule 184 applies the following rule set "Fig. 2".

\begin{tabular}{|c|c|c|c|c|c|c|c|c|}
\hline Current state & 111 & 110 & 101 & 100 & 011 & 010 & 001 & 000 \\
\hline $\begin{array}{c}\text { Next state of the } \\
\text { center cell }\end{array}$ & 1 & 0 & 1 & 1 & 1 & 0 & 0 & 0 \\
\hline
\end{tabular}

Fig. 2. Rule 184 ruleset.

The "184" in Rule 184 is the decimal representation of the bottom line "10111000" in "Fig. 2". When applying rule 184, the speed of the car is constant and does not take acceleration into account; it can go forward by one cell in one step; it will go forward if there is no car in one previous cell, and stay in the current cell if there is a car in one previous cell.

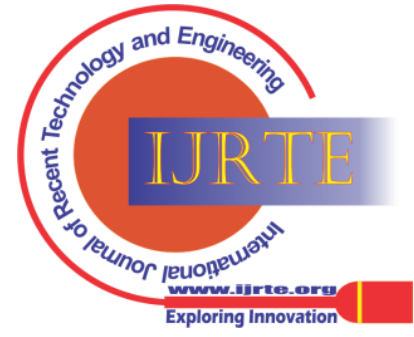


A traffic jam can be represented by dividing the road into cells and letting the steps elapse simultaneously.

\section{Basic diagram of Rule 184}

The basic theoretical diagram of Rule 184 is shown below. When the density is $p$ and the traffic volume is $q$, the basic diagram can be represented as shown in "Fig. 3".

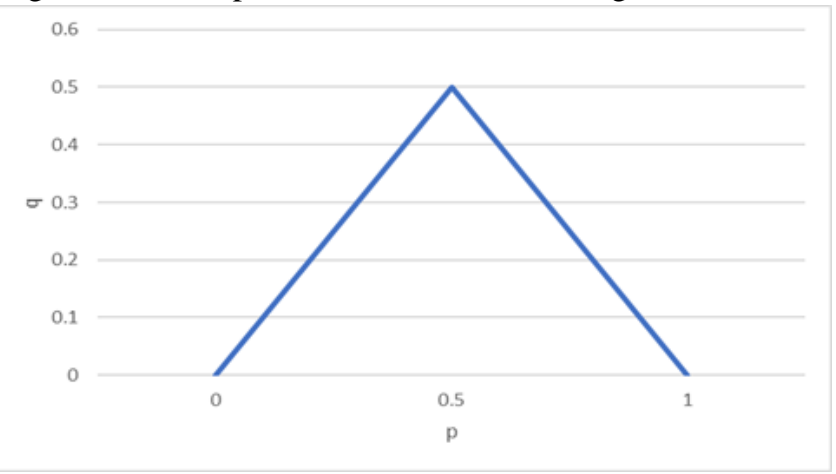

Fig. 3. Basic diagram of Rule 184.

In the basic diagram, between 0 and 0.5 , the density increases as the number of cars increases, and the traffic volume increases proportionally. In this region, all the cars in the cell are in a non-congested state where they are advancing without stopping due to the passage of steps. However, when the density exceeds 0.5 , the traffic volume is decreasing. This region is a congested state in which there are cars that cannot proceed with the passage of steps.The density when it changes from non-congested state to congested state, that is, the critical density in Rule 184, is 0.5 .

\section{TRAFFIC SIMULATION}

This section describes the target road of the traffic simulator created in the research, the reason for its setting, and the contents of the simulation.

\section{A. Simulation target}

The simulation target is the Kakuto Tunnel. The Kakuto Tunnel is a road tunnel located on the border between Ebino City, Miyazaki Prefecture and Hitoyoshi City, Kumamoto Prefecture. It is located between the Ebino IC and the Hitoyoshi Kuma Smart IC on the Kyushu Expressway, and is classified as a long tunnel because it is more than $5 \mathrm{~km}$ long [1]-[4].

The specifications are as follows.

- Length: upstream: 6255m, downstream: 6264m

- Number of lanes: 2 lanes on each side

- Design speed: $80 \mathrm{~km} / \mathrm{h}$

In this study, the upstream Kakudo Tunnel is used as the simulation target.

\section{B. Reasons for the simulation setting}

In a tunnel, the driver's speed is likely to decrease due to the sudden darkening of the driver's vision and the feeling of pressure [2]-[4]. Before entering a tunnel, the lights are turned on to indicate to following vehicles that the brakes are not being used. However, now that automatic lights are mandatory, many cars turn on their lights after entering the tunnel. This turning on of the lights in the tunnel can lead to traffic jams as the following vehicles feel that the car in front of them is using the brakes.

The volume of traffic in the Kakudo Tunnel is very high on holidays, and traffic jams often occur. In addition, the capacity of the tunnel section is insufficient for the traffic volume. In order to improve the traffic volume, it is necessary to increase the maximum speed of the expressway and add more lanes. In the case of raising the maximum speed, it was implemented on the New Tomei Expressway, but there was almost no change in speed or the number of accidents compared to before the implementation [7]. Additional lanes can be added by reducing the shoulder. Therefore, the effect of adding lanes on traffic will be studied by simulation.

\section{Description of the Simulation}

In this study, a two-lane simulation using cellular automaton is performed first, and the accuracy of the produced simulator is shown by comparing the results of the two-lane simulation with the measured data of the Kakudo Tunnel. The percentage of traffic jam occurrence is used for comparison.

A cellular automaton model is used in the simulation. Since the cellular automaton model does not take acceleration into account, it assumes a vehicle speed of 80 $\mathrm{km} / \mathrm{h}$ constant or less than $80 \mathrm{~km} / \mathrm{h}$, which is the speed limit of the road to be simulated. The data obtained from the simulation is the traffic volume of vehicles exiting the tunnel and the number of times traffic jams occur. The traffic volume results are divided into tiers of 50 cars each, and the number of times is recorded for each tier.

The number of cells is 1000 per lane, and the initial position of the cars is random. The inflow rate of cars is randomly varied between $60 \%$ and $70 \%$. The number of simulations is 100. Finally, a three-lane simulation is conducted under the same conditions, and by comparing the results of the three-lane simulation with the measured data, it is examined whether increasing the number of lanes is an appropriate method of eliminating or alleviating traffic congestion.

\section{IMPLEMENTED PROGRAM}

The program used in this study was Microsoft Visual Studio Community 2019. the simulator was created using a program in $\mathrm{C}$ language.

\section{A. Representation of Roads and Cars}

The appearance of a traffic simulation varies greatly depending on the model applied. When simulating with " 1 " and "0", it is difficult to distinguish between roads and cars. This may result in incorrect simulation results. Therefore, we used the symbol "*" for cars and blank spaces for roads to make them easier to see.

\section{B. Addition of lanes}

Rule 184 is suitable for simulations on highways with one lane in each direction. Most actual highways have two or more lanes; we have extended it to simulate on roads with two or more lanes.

\section{C. lane change}

Lane changes can be seen on real highways. With the addition of lanes, we have implemented the ability to change lanes to the driving lane and the overtaking lane.

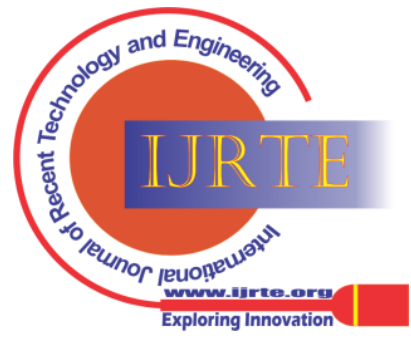




\section{Basic Study on Expressway Congestion Mitigation using Cellular Automata}

\section{Dynamic simulation representation}

Instead of displaying the traffic situation step by step, we implemented dynamic simulation so that the user can visually understand the movement of the car.

\section{EXECUTION RESULTS}

Two-lane and three-lane simulations were performed using the implemented program.

\section{A. Simulation execution screen}

The following is an example of the simulation execution screen. "Fig. 4" shows the simulation execution screen for two lanes, and "Fig. 5" shows the simulation execution screen for three lanes. In this example, 100 cells are used per lane.

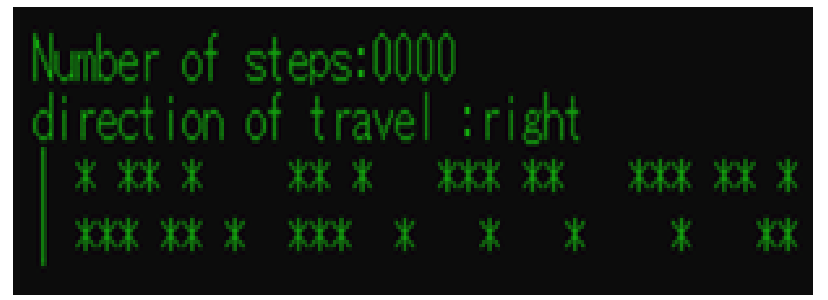

Fig. 4. Part of the two-lane simulation execution screen.

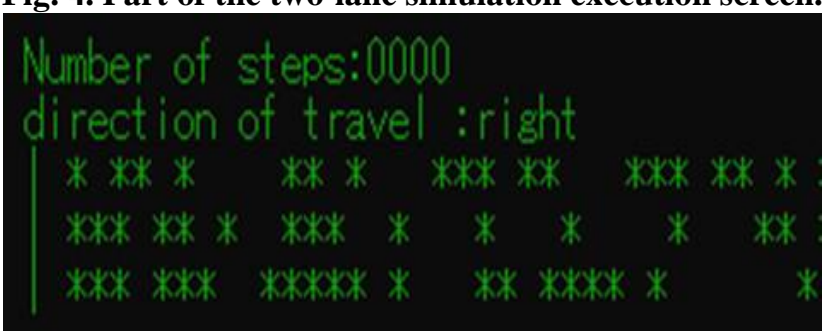

Fig. 5. Part of the 3-lane simulation execution screen.

\section{B. Ratio of Traffic Jam Occurrence}

The method of calculating the percentage of traffic congestion in this study is explained below. The traffic volume was divided into tiers of 50 vehicles each, and the number of times the traffic volume was recorded. The traffic volume count was divided by the number of times traffic congestion occurred during the hierarchy to calculate the congestion rate.

\section{Simulation Results}

The simulation results are shown in the figures below.

"Fig. 6" compares the congestion rate of the Kakuto Tunnel with the two-lane simulation using the cellular automaton model. "Fig. 7" compares the rate of congestion in the Kakuto Tunnel with a three-lane simulation using the cellular automaton model. The data used for the comparison was calculated from the actual measured traffic volume for the past two years [8]. The results of the two-lane simulation showed a higher overall rate of traffic congestion compared to the actual measurement data. The results of the two-lane simulation were similar to the actual data up to about 1,200 vehicles, but the percentage increased sharply after 1,200 vehicles; both the two-lane simulation results and the actual data showed that the percentage of congestion was $100 \%$ in the 1350 to 1,400 vehicle range.

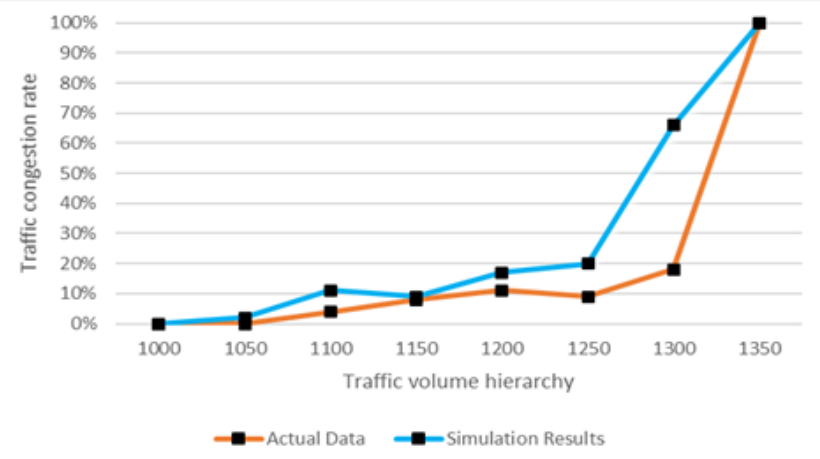

Fig. 6. Comparison of two-lane simulation results with measured data.

The three-lane simulation showed an overall reduction in traffic congestion. However, depending on the traffic volume tier, the percentage of congestion was higher than the actual measured data in some areas. In addition, the results exceeded $50 \%$ in the tiers of 1350 to 1400 vehicles.

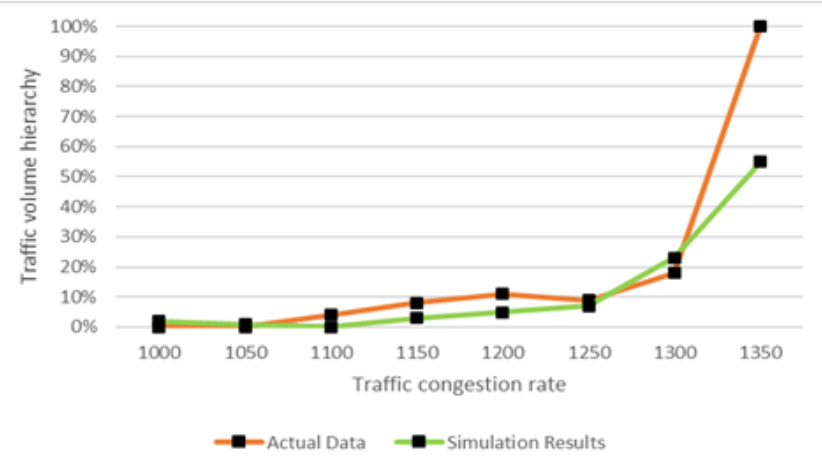

Fig. 7. Comparison of three-lane simulation results with measured data.

"Fig. 8" combines the results of "Fig. 6" and "Fig. 7".

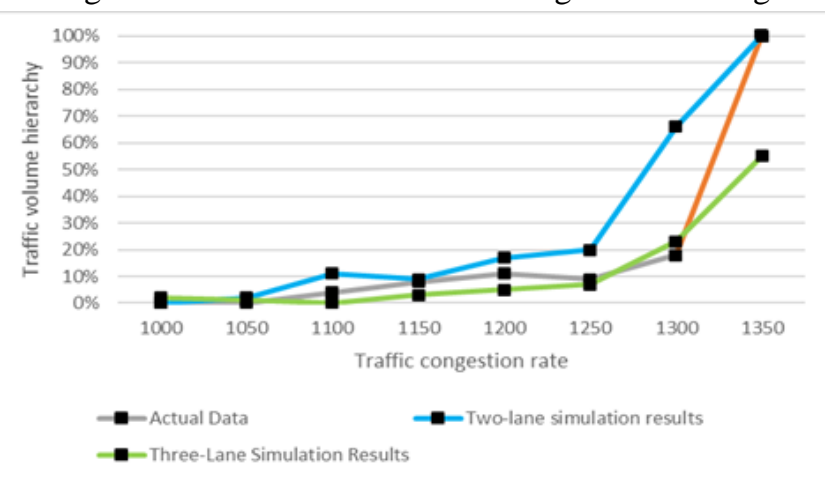

Fig. 8. Comparison of simulation results with measured data.

\section{CONSIDERATION}

Using the cellular automaton model, we were able to represent two- and three-lane traffic jams and implement a simple dynamic simulation. By actually running the simulation, we were able to confirm smooth traffic flow during non-congested conditions and stagnation of vehicles during congested conditions. However, it was difficult to set the parameters for generating traffic jams, and the users themselves had to change the parameters while actually conducting the simulation.

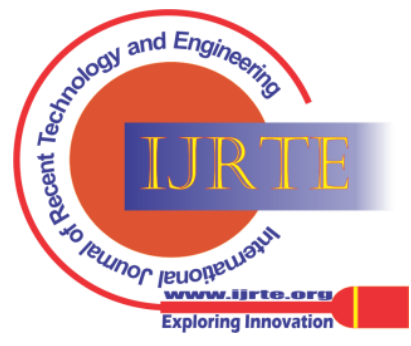


The results of the two-lane simulation showed a larger error from the actual data as the traffic volume increased. This may be related to the fact that there are many cars that exceed the speed limit in reality. The simulation using the cellular automaton model does not take acceleration into account, and assumes cars with a constant speed limit or below the speed limit. We believe that the difference in speed between cars running above and below the speed limit causes more cars to brake, which is then transmitted to the rear of the convoy, causing an explosive traffic jam at a certain traffic volume. The results of the three-lane simulation showed a lower overall rate of traffic jam occurrence compared to the actual measurement data. However, the fact that the percentage exceeded $50 \%$ in the tiers with traffic volumes between 1,350 and 1,400 vehicles indicates that increasing the number of lanes in the Kakudo Tunnel is not an appropriate method of reducing congestion. Rule 184, which is generally used in simulations of one-lane highways, was extended to two and three lanes. This allowed us to perform dynamic simulations, including lane changes. However, since the initial state of the car is randomly placed, traffic jams may occur at the initial state. In the future, we would like to make it possible to set the traffic volume for each lane. This time, we were able to limit the simulation to the Kakudo Tunnel, which has a straight road shape. In the future, it will be necessary to examine roads with gentle curves, sharp curves, and uphill slopes, all of which have the potential to reduce vehicle speed. For curves, I think it is possible to express the decrease in speed by including probability in the cellular automaton model. The cellular automaton model has the advantage of being computationally inexpensive, so we need to consider how to take advantage of this advantage even if we include probability. Furthermore, weather conditions were not taken into account in the present simulation. Weather conditions have a significant impact on the braking stopping distance. When it rains or snows, the distance between braking and stopping the car may be longer because of the reduced friction with the tires. I believe that simulations that take weather conditions into account will lead to a more realistic reproduction of road traffic. Currently, the development of self-driving cars by automobile manufacturers is in progress. It is predicted that self-driving cars will be driving on highways in the future. We would like to work on creating simulations assuming that automated vehicles and human-driven vehicles will run together. We would like to make efforts to improve the simulation by considering the above mentioned points in the future.

\section{CONCLUSION}

The purpose of this study was to represent traffic congestion in a long tunnel through traffic simulation, and to examine whether increasing the number of lanes is an appropriate way to solve or alleviate traffic congestion. To represent the traffic congestion, we used rule 184 of the commonly used cellular automaton model. In addition, as mentioned in the consideration, this was a simple simulation, and there are many aspects that have not yet been implemented, such as curves, uphills, and weather effects. There are still many issues to be solved for practical traffic simulation.

In addition, we need to increase the functionality of the cellular automaton model by focusing on its advantage of low computational complexity. In the future, we would like to study the driving characteristics of people and the causes of traffic congestion so that we can handle not only highway simulations but also traffic simulations for general roads. In simulate vehicle movements and roads with complex shapes, including the improvement measures mentioned above, so that we can contribute to the fundamental elimination and mitigation of traffic congestion through more realistic traffic simulations.

\section{REFERENCES}

1. Curriculum Subcommittee Science Working Group "Materials on Science" , February 5, 2016. [Online]. Available:

2. https://www.mext.go.jp/b_menu/shingi/chukyo/chukyo3/060/siryo/_ _icsFiles/afieldfile/2016/02/19/1367079_7.pdf

3. Ministry of Land, Infrastructure, Transport and Tourism: Statistical Information. [Online]. Available:

4. https://www.mlit.go.jp/

5. West Nippon Expressway Company Limited: Road Traffic Information Website. [Online]. Available:

6. http://www.w-nexco.co.jp/

7. A. Tomoeda, Cellular automaton as a mathematical model for analyzing "traffic jam" and its application. [Online]. Available:

8. https://www.jstage.jst.go.jp/article/bjsiam/22/1/22_KJ00008019600/ _pdf/-char/ja

9. East Nippon Expressway Company Limited: Measures against traffic jam on expressway. [Online]. Available:

10. https://www.e-nexco.co.jp/activity/safety/detail_07.html

11. Miyazaki Prefecture: Miyazaki's Road 2020. [Online]. Available:

12. https://www.pref.miyazaki.lg.jp/shakaikiban/kotsu/doro-hashi/doro/i ndex.html

13. T Tamaki, R Yasue, E Kita, Traffic Simulation of Motorway by Cellular Automata, Transactions of Information Processing Society of Japan Vol.46, 2005.

14. National Police Agency Web site: Expressway. [Online]. Available:

15. https://www.npa.go.jp/bureau/traffic/highway/index.html

16. Ministry of Land, Infrastructure, Transport and Tourism, Road Bureau: Current status and transition of road traffic from the Road Traffic Census. [Online]. Available:

17. https://www.mlit.go.jp/road/ir/ir-data/data_shu.html

\section{AUTHORS PROFILE}

Kodai Miyamoto, is a master student at Department of Computer Science and System Engineering, University of Miyazaki. His current research topic is a fundamental study on educational support using VR technology.

Taketo Kamasaka, is a master student at Departmen of Computer Science and System Engineering, University of Miyazaki. His current research interests are computer graphics, entertainment computing, and so on.

Makoto Sakamoto, received the Ph.D. degree in computer science and systems engineering from Yamaguchi University in 1999. He is presently a professor in the Faculty of Engineering, University of Miyazaki. His first interests lay in hydrodynamics and time series analysis, especially the directional wave spectrum. He is a theoretical computer scientist, and 2. his current main research interests are automata theory, languages and computation. 


\section{Basic Study on Expressway Congestion Mitigation using Cellular Automata}

He is also interested in digital geometry, digital image processing, computer vision, computer graphics, virtual reality, augmented reality, entertainment computing, complex systems and so forth. He has published many research papers in that area. His articles have appeared in journals such as Information Sciences, Pattern Recognition and Artificial Intelligence, WSEAS, AROB, ICAROB, SJI,IEEE and IEICE (Japan), and proceedings of conferences such as Parallel Image Processing and Analysis, SCI, WSEAS, AROB and ICAROB.

Tsunehiro Yoshinaga, received his B.S. degree in mathematics and Ph.D degree in computer science and systems engineering from Yamaguchi University in 1982 and 1996, respectively. Since 1982, he has been with Tokuyama College of Technology. He is presently a Professor in the Department of Computer Science and Electronics Engineering. His current research interests are automata theory and computational complexity.

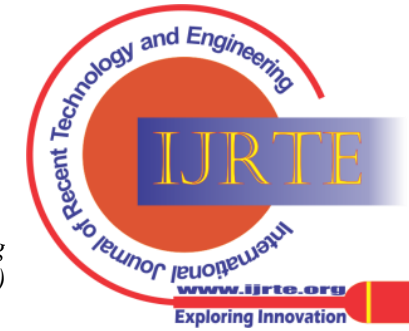

